PROCEEDINGS OF THE

AMERICAN MATHEMATICAL SOCIETY

Volume 139, Number 1, January 2011, Pages 259-271

S 0002-9939(2010)10479-3

Article electronically published on August 6, 2010

\title{
REGULAR METHODS OF SUMMABILITY ON TREE-SEQUENCES IN BANACH SPACES
}

\author{
COSTAS POULIOS
}

(Communicated by Nigel J. Kalton)

\begin{abstract}
Suppose that $X$ is a Banach space, $\left\langle a_{i j}\right\rangle$ is a regular method of summability and $\left(x_{s}\right)_{s \in S}$ is a bounded sequence in $X$ indexed by the dyadic tree $S$. We prove that there exists a subtree $S^{\prime} \subseteq S$ such that: either (a) for any chain $\beta$ of $S^{\prime}$ the sequence $\left(x_{s}\right)_{s \in \beta}$ is summable with respect to $\left\langle a_{i j}\right\rangle$ or (b) for any chain $\beta$ of $S^{\prime}$ the sequence $\left(x_{s}\right)_{s \in \beta}$ is not summable with respect to $\left\langle a_{i j}\right\rangle$. Moreover, in case (a) we prove the existence of a subtree $T \subseteq S^{\prime}$ such that if $\beta$ is any chain of $T$, then all the subsequences of $\left(x_{s}\right)_{s \in \beta}$ are summable to the same limit. In case (b), provided that $\left\langle a_{i j}\right\rangle$ is the Cesàro method of summability and that for any chain $\beta$ of $S^{\prime}$ the sequence $\left(x_{s}\right)_{s \in \beta}$ is weakly null, we prove the existence of a subtree $T \subseteq S^{\prime}$ such that for any chain $\beta$ of $T$ any spreading model for the sequence $\left(x_{s}\right)_{s \in \beta}$ has a basis equivalent to the usual $l_{1}$-basis. Finally, we generalize the theory of spreading models to tree-sequences. This also allows us to improve the result of case (b).
\end{abstract}

\section{INTRODUCTION}

An infinite matrix $\left\langle a_{i j}\right\rangle_{i, j \in \mathbb{N}}$ of real numbers is called a regular method of summability if, given a sequence $\left(x_{i}\right)_{i \in \mathbb{N}}$ of elements of a Banach space $X$ converging to $x \in X$, the sequence $\left(x_{i}^{\prime}\right)$, where $x_{i}^{\prime}=\sum_{j=1}^{\infty} a_{i j} x_{j}$, is well-defined and also converges to $x$. A sequence $\left(x_{i}\right)$ in a Banach space is called summable with respect to $\left\langle a_{i j}\right\rangle$ if the sequence $\left(x_{i}^{\prime}\right)$ is well-defined and converges. The following proposition characterizes the regular methods of summability (see [3]).

Proposition 1.1. An infinite matrix $\left\langle a_{i j}\right\rangle$ is a regular method of summability if and only if the following conditions hold:

(1) $\sup _{i} \sum_{j=1}^{\infty}\left|a_{i j}\right|<\infty$,

(2) $\lim _{i \rightarrow \infty} a_{i j}=0$ for every $j$ and

(3) $\lim _{i \rightarrow \infty} \sum_{j=1}^{\infty} a_{i j}=1$.

The following theorem, concerning summability of bounded sequences in Banach spaces, is due to P. Erdös and M. Magidor [4. Its proof is based on the GalvinPrikry theorem [5] (see also [9]).

Theorem 1.1. Suppose that $X$ is a Banach space, $\left\langle a_{i j}\right\rangle$ is a regular method of summability and $\left(x_{i}\right)$ is a bounded sequence in $X$. Then there exists a subsequence $\left(y_{i}\right)$ of $\left(x_{i}\right)$ such that: either

Received by the editors December 11, 2009 and, in revised form, February 16, 2010 and March 1, 2010.

2010 Mathematics Subject Classification. Primary 40C05, 46B99; Secondary 05D10, 05C55.

(C)2010 American Mathematical Society

Reverts to public domain 28 years from publication 
(1) all subsequences of $\left(y_{i}\right)$ are summable to a common limit with respect to $\left\langle a_{i j}\right\rangle$ or

(2) no subsequence of $\left(y_{i}\right)$ is summable with respect to $\left\langle a_{i j}\right\rangle$.

Moreover, under some additional hypotheses, H. P. Rosenthal [7] proved the following result, concerning the second case of the previous theorem.

Theorem 1.2. Let $\left\langle a_{i j}\right\rangle$ be the Cesàro method of summability and let $\left(x_{i}\right)$ be a weakly null sequence. Suppose that no subsequence of $\left(x_{i}\right)$ is summable with respect to $\left\langle a_{i j}\right\rangle$. Then there are a subsequence $\left(y_{i}\right)$ of $\left(x_{i}\right)$ and $a \delta>0$ such that for any $k \in \mathbb{N}$, any $k \leq n_{1}<n_{2}<\ldots<n_{2^{k}}$ and any scalars $c_{1}, c_{2}, \ldots, c_{2^{k}}$,

$$
\left\|\sum_{i=1}^{2^{k}} c_{i} y_{n_{i}}\right\| \geq \delta \sum_{i=1}^{2^{k}}\left|c_{i}\right| .
$$

Throughout this paper $S$ denotes the standard dyadic tree, that is, the set of all finite sequences in $\{0,1\}$, including the empty sequence denoted by $\emptyset$. Elements $s \in S$ are called nodes. If $s$ is a node and $s \in\{0,1\}^{n}$, we say that $s$ is on the $n$-th level of $S$. We denote the level of a node $s$ by $\operatorname{lev}(s)$. The initial segment partial ordering on $S$ is denoted by $\leq$ and we write $s<s^{\prime}$ if $s \leq s^{\prime}$ and $s \neq s^{\prime}$. If $s \leq s^{\prime}$, we say that $s^{\prime}$ is a follower of $s$, while if $s, s^{\prime}$ are nodes such that neither $s \leq s^{\prime}$ nor $s^{\prime} \leq s$, then $s$ and $s^{\prime}$ are called noncomparable.

A partially ordered set $T$ is called a dyadic tree if it is order isomorphic to $(S, \leq)$. Thus a subtree of $S$ is any subset $S^{\prime}$ of $S$ which has a single minimal element and any element of $S^{\prime}$ has exactly two immediate successors. In the following, by a tree we shall mean a dyadic tree. If $T$ is a tree, a chain of $T$ is an infinite linearly ordered subset of $T$. The set of all chains of $T$ is denoted by $\mathcal{C}(T)$.

The set $\mathcal{C}(T)$ is endowed with the relative topology of the product topology of $\mathcal{P}(T)$. A subbasis of this topology consists of the sets $U(t)=\{\beta \in \mathcal{C}(T) \mid t \in \beta\}$ and $V(t)=\{\beta \in \mathcal{C}(T) \mid t \notin \beta\}$ when $t$ varies over the elements of $T$. Clearly the sets $U(t), V(t)$ are open and closed. It is also known (see [8]) that $\mathcal{C}(T)$ is a $G_{\delta}$ subset of $\mathcal{P}(T)$. Therefore $\mathcal{C}(T)$ is a Polish space (see [6]).

Considering on $\mathcal{C}(T)$ the topology described above, J. Stern [8] proved the following Ramsey-type theorem for the dyadic tree.

Theorem 1.3. Let $T$ be a tree and let $\mathcal{A} \subset \mathcal{C}(T)$ be an analytic set of chains. There exists a subtree $T^{\prime}$ of $T$ such that either $\mathcal{C}\left(T^{\prime}\right) \subset \mathcal{A}$ or $\mathcal{C}\left(T^{\prime}\right) \cap \mathcal{A}=\emptyset$.

In the present paper we consider bounded sequences $\left(x_{s}\right)_{s \in S}$ of elements of a Banach space indexed by $S$. Using Stern's theorem instead of the Galvin-Prikry theorem, we study the summability of the sequences $\left(x_{s}\right)_{s \in \beta}$ for $\beta \in \mathcal{C}(S)$. In this direction, we prove the following dichotomy result.

Theorem 1.4. Suppose that $X$ is a Banach space, $\left\langle a_{i j}\right\rangle$ is a regular method of summability and $\left(x_{s}\right)_{s \in S}$ is a bounded sequence of elements in $X$. There exists a subtree $S^{\prime}$ of $S$ such that: either

(1) for any chain $\beta \in \mathcal{C}\left(S^{\prime}\right)$, the sequence $\left(x_{s}\right)_{s \in \beta}$ is summable with respect to $\left\langle a_{i j}\right\rangle$ or

(2) for no chain $\beta \in \mathcal{C}\left(S^{\prime}\right)$, the sequence $\left(x_{s}\right)_{s \in \beta}$ is summable with respect to $\left\langle a_{i j}\right\rangle$. 
In Section 3 we assume that the first case happens and we prove the existence of a subtree $T$ of $S^{\prime}$ such that if $\beta$ is any (maximal) chain of $T$, then all the subsequences of $\left(x_{s}\right)_{s \in \beta}$ are summable to the same limit. Moreover, it is proved that for any $\epsilon>0$ we can find a subset $B \subset X$ with $\operatorname{diam}(B) \leq \epsilon$ and a further subtree such that for every chain $\beta$ the sequence $\left(x_{s}\right)_{s \in \beta}$ is summable to a point of the set $B$.

In Section 4 we assume that the second case of Theorem 1.4 happens. Furthermore, we suppose that $\left\langle a_{i j}\right\rangle$ is the Cesàro method of summability and that for any chain $\beta$ of $S^{\prime}$ the sequence $\left(x_{s}\right)_{s \in \beta}$ is weakly null. Then we prove the counterpart of Rosenthal's theorem. There are a subtree $T$ of $S^{\prime}$ and a $\delta>0$ with the following property: for any chain $\beta=\left\{s_{1}<s_{2}<\ldots\right\}$ of $T$, any $k \in \mathbb{N}$ and $k \leq n_{1}<n_{2}<\ldots<n_{2^{k}}$ and any scalars $c_{1}, c_{2}, \ldots, c_{2^{k}}$,

$$
\left\|\sum_{i=1}^{2^{k}} c_{i} x_{s_{n_{i}}}\right\| \geq \delta \sum_{i=1}^{2^{k}}\left|c_{i}\right| .
$$

As a corollary, we obtain that for any chain $\beta$ of $T$, any spreading model for the sequence $\left(x_{s}\right)_{s \in \beta}$ has a basis equivalent to the usual $l_{1}$-basis.

Finally, in Section 5 we generalize the theory of spreading models to treesequences. More precisely, we assume that $\left(x_{s}\right)_{s \in S}$ is a normalized sequence and $\left(\epsilon_{i}\right)$ is a sequence of positive real numbers converging to 0 . Then we find a subtree $T$ of $S$ such that for any chain $\beta=\left\{s_{1}<s_{2}<\ldots\right\}$ of $T$, any $k \in \mathbb{N}$, any $\left(a_{i}\right)_{i=1}^{2^{k}} \subset[-1,1]$, any $k \leq n_{1}<\ldots<n_{2^{k}}$ and any $k \leq m_{1}<\ldots<m_{2^{k}}$,

$$
\left|\left\|\sum_{i=1}^{2^{k}} a_{i} x_{s_{n_{i}}}\right\|-\left\|\sum_{i=1}^{2^{k}} a_{i} x_{s_{m_{i}}}\right\|\right| \leq \epsilon_{k} .
$$

This result also allows us to improve the corollary of Section 4

\section{The MAIN Dichotomy}

This section is devoted to the proof of Theorem 1.4. First, we state the following lemma, whose proof is straightforward.

Lemma 2.1. Let $\left(x_{s}\right)_{s \in S}$ be a bounded sequence in the Banach space $X$. For every $i \in \mathbb{N}$ we define the function $f_{i}: \mathcal{C}(S) \rightarrow X$ by

$$
\beta=\left\{s_{1}<s_{2}<\ldots\right\} \mapsto f_{i}(\beta)=\sum_{j=1}^{\infty} a_{i j} x_{s_{j}} .
$$

Then $f_{i}$ is continuous.

Proof of Theorem 1.4. Consider the set

$$
\mathcal{A}=\left\{\beta \in \mathcal{C}(S) \mid\left(x_{s}\right)_{s \in \beta} \text { is summable with respect to }\left\langle a_{i j}\right\rangle\right\} .
$$

Claim. The set $\mathcal{A}$ is a Borel subset of $\mathcal{C}(S)$.

Indeed, observe that

$$
\begin{aligned}
\beta=\left\{s_{1}<s_{2}<\ldots\right\} \in \mathcal{A} & \Leftrightarrow\left(x_{s}\right)_{s \in \beta} \text { is summable with respect to }\left\langle a_{i j}\right\rangle \\
& \Leftrightarrow \text { the sequence }\left(y_{i}\right), y_{i}=\sum_{j=1}^{\infty} a_{i j} x_{s_{j}}, \text { converges in } X \\
& \Leftrightarrow\left(y_{i}\right) \text { is a Cauchy sequence } \\
& \Leftrightarrow\left(\forall q \in \mathbb{Q}^{+} \exists i_{0} \in \mathbb{N}\right)\left[\left(\forall n, m \geq i_{0}\right)\left(\left\|y_{n}-y_{m}\right\|<q\right)\right] .
\end{aligned}
$$


Therefore,

$$
\mathcal{A}=\bigcap_{q \in \mathbb{Q}^{+}} \bigcup_{i_{0} \in \mathbb{N}} \bigcap_{n, m \geq i_{0}} \mathcal{D}_{q, n, m}
$$

where

$$
\mathcal{D}_{q, n, m}=\left\{\beta=\left\{s_{1}<s_{2}<\ldots\right\} \in \mathcal{C}(S) \mid\left\|\sum_{j=1}^{\infty} a_{n j} x_{s_{j}}-\sum_{j=1}^{\infty} a_{m j} x_{s_{j}}\right\|<q\right\} .
$$

By Lemma 2.1 the set $\mathcal{D}_{q, n, m}$ is open, being the inverse image of the open ball $\{x \in X \mid\|x\|<q\}$ in $X$ by the continuous function $f_{n}-f_{m}$ (here $f_{n}$ and $f_{m}$ are as in Lemma 2.11). Hence the set $\mathcal{A}$ is Borel.

By Stern's theorem, there is a subtree $S^{\prime}$ of $S$ such that either $\mathcal{C}\left(S^{\prime}\right) \subset \mathcal{A}$ or $\mathcal{C}\left(S^{\prime}\right) \cap \mathcal{A}=\emptyset$. That is, either:

(1) for any chain $\beta$ of $S^{\prime}$, the sequence $\left(x_{s}\right)_{s \in \beta}$ is summable with respect to $\left\langle a_{i j}\right\rangle$ or

(2) for no chain $\beta$ of $S^{\prime}$, the sequence $\left(x_{s}\right)_{s \in \beta}$ is summable with respect to $\left\langle a_{i j}\right\rangle$.

\section{The CASe Where All the Sequences $\left(x_{s}\right)_{s \in \beta}$ ARE Summable}

In this section we assume that the first case of Theorem 1.4 happens. Then we find further subtrees $T$ such that the limits to which the sequences $\left(x_{s}\right)_{s \in \beta}$, $\beta \in \mathcal{C}(T)$, are summable have some nice properties. The first result in this direction is the following.

Theorem 3.1. Let $\left(x_{s}\right)_{s \in S}$ be a bounded sequence in the Banach space $X$ and $\left\langle a_{i j}\right\rangle$ be a regular method of summability. We assume that for each chain $\beta \in \mathcal{C}(S)$ the sequence $\left(x_{s}\right)_{s \in \beta}$ is summable with respect to $\left\langle a_{i j}\right\rangle$. Then there is a subtree $T$ of $S$ such that if $\beta$ is any chain of $T$, then for each subchain $\alpha \subset \beta$ the sequences $\left(x_{s}\right)_{s \in \alpha},\left(x_{s}\right)_{s \in \beta}$ are summable to the same limit.

Proof. Consider the following subset of $\mathcal{C}(S)$ :

$\mathcal{F}=\left\{\beta \in \mathcal{C}(S) \mid\right.$ for each $\alpha \in \mathcal{C}(S)$ with $\alpha \subset \beta$, the sequences $\left(x_{s}\right)_{s \in \alpha},\left(x_{s}\right)_{s \in \beta}$ are summable to the same limit\}.

Claim. The set $\mathcal{F}$ is coanalytic.

In particular, we prove that the complement of $\mathcal{F}$, that is, the set $\{\beta \in \mathcal{C}(S) \mid$ there is $\alpha \in \mathcal{C}(S), \alpha \subset \beta$, such that the sequences $\left(x_{s}\right)_{s \in \alpha},\left(x_{s}\right)_{s \in \beta}$ are not summable to the same limit $\}$, is analytic.

As mentioned in the introduction, $\mathcal{C}(S)$ is a Polish space. We now consider the space $\mathcal{C}(S) \times \mathcal{C}(S)$, endowed with the product topology, and the following subset:

$$
\mathcal{A}=\left\{(\beta, \alpha) \in \mathcal{C}(S) \times \mathcal{C}(S) \mid \alpha \subset \beta \text { and the sequences }\left(x_{s}\right)_{s \in \alpha},\left(x_{s}\right)_{s \in \beta}\right. \text { are not }
$$
summable to the same limit\}.

Clearly,

$$
\mathcal{A}=\mathcal{A}_{1} \cap \mathcal{A}_{2}
$$

where

$\mathcal{A}_{1}=\{(\beta, \alpha) \in \mathcal{C}(S) \times \mathcal{C}(S) \mid \alpha \subset \beta\}$,

$\mathcal{A}_{2}=\left\{(\beta, \alpha) \in \mathcal{C}(S) \times \mathcal{C}(S) \mid\left(x_{s}\right)_{s \in \alpha},\left(x_{s}\right)_{s \in \beta}\right.$ are not summable to the same limit $\}$. 
First, we observe that $\mathcal{A}_{1}$ is a closed set. Indeed, it is enough to write down the following characterization of the set $\mathcal{A}_{1}$ :

$$
\begin{aligned}
(\beta, \alpha) \in \mathcal{A}_{1} & \Leftrightarrow \alpha \subset \beta \\
& \Leftrightarrow(\forall s \in S)[s \in \alpha \Rightarrow s \in \beta] .
\end{aligned}
$$

Next, we argue that the set $\mathcal{A}_{2}$ is Borel. Indeed, we have:

$$
\begin{aligned}
(\beta, \alpha) \in \mathcal{A}_{2} & \Leftrightarrow\left(x_{s}\right)_{s \in \alpha},\left(x_{s}\right)_{s \in \beta} \text { are not summable to the same limit } \\
& \Leftrightarrow\left(\exists q \in \mathbb{Q}^{+} \exists i_{0} \in \mathbb{N}\right)\left[\left(\forall i \geq i_{0}\right)\left(\left\|\sum_{j=1}^{\infty} a_{i j} x_{t_{j}}-\sum_{j=1}^{\infty} a_{i j} x_{s_{j}}\right\|>q\right)\right],
\end{aligned}
$$

where $\alpha=\left\{t_{1}<t_{2}<\ldots\right\}, \beta=\left\{s_{1}<s_{2}<\ldots\right\}$. Therefore,

$$
\mathcal{A}_{2}=\bigcup_{q \in \mathbb{Q}^{+}} \bigcup_{i_{0} \in \mathbb{N}} \bigcap_{i \geq i_{0}} \mathcal{G}_{q, i}
$$

where

$$
\mathcal{G}_{q, i}=\left\{(\beta, \alpha) \in \mathcal{C}(S) \times \mathcal{C}(S) \mid\left\|\sum_{j=1}^{\infty} a_{i j} x_{t_{j}}-\sum_{j=1}^{\infty} a_{i j} x_{s_{j}}\right\|>q\right\} .
$$

The set $\mathcal{G}_{q, i}$ is open, being the inverse image of the open set $\{x \in X \mid\|x\|>q\}$ by the continuous function $\mathcal{C}(S) \times \mathcal{C}(S) \rightarrow X$ with $(\beta, \alpha) \mapsto f_{i}(\beta)-f_{i}(\alpha)$. Hence, the set $\mathcal{A}_{2}$ is Borel.

Therefore, the set $\mathcal{A}=\mathcal{A}_{1} \cap \mathcal{A}_{2}$ is a Borel subset of $\mathcal{C}(S) \times \mathcal{C}(S)$. To complete the proof of the claim, we consider the projection $\mathcal{C}(S) \times \mathcal{C}(S) \rightarrow \mathcal{C}(S)$ with $(\beta, \alpha) \mapsto \beta$ and observe that the image of the Borel set $\mathcal{A}$ by this projection is exactly the set $\mathcal{C}(S) \backslash \mathcal{F}$. Consequently, the set $\mathcal{C}(S) \backslash \mathcal{F}$ is analytic.

We now apply Theorem 1.3. Since $\mathcal{F}$ is coanalytic, we find a subtree $T$ of $S$ such that either $\mathcal{C}(T) \subset \mathcal{F}$ or $\mathcal{C}(T) \cap \mathcal{F}=\emptyset$. That is, one of the following possibilities holds:

(1) for any chain $\beta \in \mathcal{C}(T)$ and for any subchain $\alpha \subset \beta$, the sequences $\left(x_{s}\right)_{s \in \beta}$, $\left(x_{s}\right)_{s \in \alpha}$ are summable to the same limit;

(2) for any chain $\beta \in \mathcal{C}(T)$ there is a subchain $\alpha \subset \beta$ such that $\left(x_{s}\right)_{s \in \beta},\left(x_{s}\right)_{s \in \alpha}$ are not summable to the same limit.

Thus, what we need is to exclude the second possibility. Assume it happens and let $\beta$ be any chain of $T$. Applying the Erdös-Magidor theorem, we find a subchain $\beta^{\prime} \subset \beta$ such that all the subsequences of $\left(x_{s}\right)_{s \in \beta^{\prime}}$ are summable to the same limit. Therefore, $\beta^{\prime}$ is a chain of $T$ which does not satisfy condition (2). Thus, we have reached a contradiction.

In general, it is not possible to find a subtree $T$ of $S$ and an element $x \in X$ such that for each chain $\beta$ of $T$ the sequence $\left(x_{s}\right)_{s \in \beta}$ is summable to $x$. However, we can find a subset $B \subset X$ with small diameter and a subtree $T$ such that each sequence $\left(x_{s}\right)_{s \in \beta}, \beta \in \mathcal{C}(T)$, is summable to a limit which lies in $B$. This is the content of our next theorem.

Theorem 3.2. Let $\left(x_{s}\right)_{s \in S}$ and $\left\langle a_{i j}\right\rangle$ be as in the previous theorem and let $\epsilon>0$ be given. Then there are a subset $B$ of $X$ with $\operatorname{diam}(B) \leq \epsilon$ and a subtree $T \subset S$ such that for any chain $\beta$ of $T$, the sequence $\left(x_{s}\right)_{s \in \beta}$ is summable to some point of the set $B$.

For the proof we need the following lemma. 
Lemma 3.3. Let $\left(z_{j}\right)$ be a bounded sequence in the Banach space $X$ which is summable to $z$ with respect to $\left\langle a_{i j}\right\rangle$ and let $v_{1}, \ldots, v_{N} \in X$. Then the sequence $\left(v_{1}, \ldots, v_{N}, z_{N+1}, z_{N+2}, \ldots\right)$ is also summable to $z$ with respect to $\left\langle a_{i j}\right\rangle$.

Proof of Theorem [3.2, Let $Y=\overline{\operatorname{span}}\left\{x_{s} \mid s \in S\right\}$ be the closed linear span of $\left(x_{s}\right)_{s \in S}$. Then $Y$ is a separable Banach space and for any chain $\beta$ the sequence $\left(x_{s}\right)_{s \in \beta}$ is summable to a limit which lies in $Y$. Choose a countable cover $\left\{B_{n} \mid n \in\right.$ $\mathbb{N}\}$ of $Y$ consisting of open balls of radius $\epsilon / 2$. Consider the following subset of $\mathcal{C}(S)$ :

$$
\mathcal{A}=\left\{\beta \in \mathcal{C}(S) \mid\left(x_{s}\right)_{s \in \beta} \text { is summable to some point of the ball } B_{1}\right\} .
$$

Claim. $\mathcal{A}$ is a Borel subset of $\mathcal{C}(S)$.

Indeed, we have

$$
\begin{aligned}
\beta=\left\{s_{1}<s_{2}<\ldots\right\} & \in \mathcal{A} \\
\Leftrightarrow & \left(x_{s}\right)_{s \in \beta} \text { is summable to some point of the ball } B_{1} \\
\Leftrightarrow & \text { the limit of }\left(y_{i}\right), y_{i}=\sum_{j=1}^{\infty} a_{i j} x_{s_{j}}, \text { belongs to } B_{1} \\
& \Leftrightarrow\left(\exists q \in \mathbb{Q}^{+} \exists l \in \mathbb{N}\right)(\forall i \geq l)\left[\left\|\sum_{j=1}^{\infty} a_{i j} x_{s_{j}}-z\right\|<\frac{\epsilon}{2}-q\right],
\end{aligned}
$$

where $z$ is the center of the ball $B_{1}$. Therefore,

$$
\mathcal{A}=\bigcup_{q \in \mathbb{Q}^{+}} \bigcup_{l \in \mathbb{N}} \bigcap_{i \geq l} \mathcal{D}_{q, i}
$$

where

$$
\mathcal{D}_{q, i}=\left\{\beta=\left\{s_{1}<s_{2}<\ldots\right\} \in \mathcal{C}(S) \mid\left\|\sum_{j=1}^{\infty} a_{i j} x_{s_{j}}-z\right\|<\frac{\epsilon}{2}-q\right\} .
$$

By Lemma 2.1, the set $\mathcal{D}_{q, i}$ is open, being the inverse image of the open ball $\left\{x \in X \mid\|x-z\|<\frac{\epsilon}{2}-q\right\}$ by the continuous function $f_{i}$. Hence, the set $\mathcal{A}$ is Borel.

Applying Stern's theorem, we find a subtree $S_{1}$ of $S$ such that either $\mathcal{C}\left(S_{1}\right) \subset \mathcal{A}$ or $\mathcal{C}\left(S_{1}\right) \cap \mathcal{A}=\emptyset$. That is, one of the following conditions holds:

(a) for any chain $\beta$ of $S_{1}$, the sequence $\left(x_{s}\right)_{s \in \beta}$ is summable to a point in the ball $B_{1}$;

(b) for any chain $\beta$ of $S_{1}$, the sequence $\left(x_{s}\right)_{s \in \beta}$ is summable to a point outside the ball $B_{1}$.

Repeating the same argument, we find a sequence $S \supset S_{1} \supset S_{2} \supset \ldots$ of subtrees of $S$ such that for each $k$ exactly one of the following holds:

(1) for any chain $\beta$ of $S_{k},\left(x_{s}\right)_{s \in \beta}$ is summable to a point of the ball $B_{k}$;

(2) for any chain $\beta$ of $S_{k},\left(x_{s}\right)_{s \in \beta}$ is summable to a point outside the ball $B_{k}$.

Let $s_{k}$ denote the minimal element of the subtree $S_{k}$. Then we have $s_{1} \leq s_{2} \leq$ $s_{3} \leq \ldots$. Without loss of generality, we can assume that for each $k$ there are $N_{k}>k$ and elements $s_{k, 1}, s_{k, 2}, \ldots, s_{k, N_{k}}$ of $S_{k}$ such that

$$
s_{k}=s_{k, 1}<s_{k, 2}<\ldots<s_{k, N_{k}}=s_{k+1} .
$$

Claim. There is $k_{1} \in \mathbb{N}$ such that condition (1) holds for $S_{k_{1}}$. 
Indeed, let us suppose that for all $k$, condition (2) holds for $S_{k}$, that is, for any chain $\beta$ of $S_{k}$ the sequence $\left(x_{s}\right)_{s \in \beta}$ is summable to a point outside the ball $B_{k}$. Consider the chain $\beta=\left\{s_{1}<s_{2}<\ldots\right\}$. Then the sequence $\left(x_{s_{k}}\right)_{k \in \mathbb{N}}$ is summable, say to $y \in Y$. By Lemma 3.3 , the sequence

$$
\left(x_{s_{k, 1}}, \ldots, x_{s_{k, k}}, x_{s_{k+1}}, x_{s_{k+2}} \ldots\right)
$$

is also summable to $y$. Since $\left\{s_{k, 1}<\ldots<s_{k, k}<s_{k+1}<s_{k+2}<\ldots\right\}$ is a chain of $S_{k}$, we obtain that $y \notin B_{k}$. Since this happens for all $k$, we have reached a contradiction.

Therefore, there exists $k_{1} \in \mathbb{N}$ such that for any chain $\beta$ of $S_{k_{1}}$, the sequence $\left(x_{s}\right)_{s \in \beta}$ is summable to a point of the ball $B_{k_{1}}$. The choices $B=B_{k_{1}}$ and $T=S_{k_{1}}$ complete the proof.

Remark. We can prove Theorem 3.1 by repeating the argument used in Theorem 3.2. This proof has the advantage of using Borel sets instead of analytic. In the following we shall outline this proof.

The desired subtree $T$ is constructed inductively. We will indicate the first steps. By Theorem 3.2, there are a subset $B_{\emptyset}$ of $X$ with $\operatorname{diam} B_{\emptyset} \leq 1$ and a subtree $T_{\emptyset} \subset S$ such that for any chain $\beta$ of $T_{\emptyset}$, the sequence $\left(x_{s}\right)_{s \in \beta}$ is summable to some point of the set $B_{\emptyset}$. Let $t_{\emptyset}$ be the minimum element of $T_{\emptyset}$ and let $t_{0}, t_{1}$ be the nodes belonging to the first level of $T_{\emptyset}$. Then, $t_{\emptyset}$ is the minimum node of $T$ and $t_{0}, t_{1}$ complete the first level of $T$.

Now consider a countable cover of the set $B_{\emptyset}$, consisting of open balls in $B_{\emptyset}$ of radius $1 / 3$. Moreover, let $\widetilde{T}_{0}$ be the subtree of $T_{\emptyset}$ which contains the node $t_{0}$ and all its followers in the tree $T_{\emptyset}$. Repeat the proof of Theorem 3.2 to the sequence $\left(x_{s}\right)_{s \in \widetilde{T}_{0}}$ to obtain a subset $B_{0}$ of $B_{\emptyset}$ with $\operatorname{diam}\left(B_{0}\right) \leq 2 / 3$ and a subtree $T_{0} \subset \widetilde{T}_{0} \subset T_{\emptyset}$ such that, for any chain $\beta$ of $T_{0}$, the sequence $\left(x_{s}\right)_{s \in \beta}$ is summable to some point of the set $B_{0}$. Let $t_{(0,0)}, t_{(0,1)}$ be noncomparable nodes placed on the second at least level of $T_{0}$. This choice allows us to use Lemma 3.3. The nodes $t_{(0,0)}, t_{(0,1)}$ are the immediate successors of $t_{0}$ in $T$.

We inductively construct a subtree $T=\left\{t_{s} \mid s \in S\right\}$ of $S$ and a sequence $\left(B_{s}\right)_{s \in S}$ of subsets of $X$, such that the following properties hold:

(i) if $s \leq s^{\prime}$, then $B_{s} \supset B_{s^{\prime}}$;

(ii) $\operatorname{diam}\left(B_{\emptyset}\right) \leq 1$ and if $\operatorname{lev}(s)=n$, then $\operatorname{diam}\left(B_{s}\right) \leq 2 /(n+1)$;

(iii) if $\beta$ is any chain of $S$, then the sequence $\left(x_{t_{s}}\right)_{s \in \beta}$ is summable to some point of $\bigcap_{s \in \beta} B_{s}$ (by the construction and Lemma 3.3).

Clearly, for any chain $\beta \in \mathcal{C}(S), \operatorname{diam}\left(\bigcap_{s \in \beta} B_{s}\right)=0$; that is, the set $\bigcap_{s \in \beta} B_{s}$ is a singleton. Consequently, if $\alpha, \beta$ are chains of $S$ and $\alpha \subset \beta$, then $\bigcap_{s \in \alpha} B_{s}=\bigcap_{s \in \beta} B_{s}$ and the sequences $\left(x_{t_{s}}\right)_{s \in \alpha},\left(x_{t_{s}}\right)_{s \in \beta}$ are summable to the same limit. Thus, the subtree $T$ has the desired property.

\section{The Case Where no Sequence $\left(x_{s}\right)_{s \in \beta}$ IS Summable}

In this section we assume that the second case of Theorem 1.4 happens. Then we prove the following theorem, which is the counterpart of Theorem 1.2

Theorem 4.1. Let $\left(x_{s}\right)_{s \in S}$ be a bounded sequence in the Banach space X. Suppose that for any chain $\beta$ of $S$ the sequence $\left(x_{s}\right)_{s \in \beta}$ is weakly null and it is not Cesàro summable. Then there exist a subtree $T$ of $S$ and $a \delta>0$ such that for any chain 
$\beta=\left\{s_{1}<s_{2}<\ldots\right\}$ of $T$, any $k \in \mathbb{N}$ and $k \leq n_{1}<n_{2}<\ldots<n_{2^{k}}$ and any scalars $c_{1}, c_{2}, \ldots, c_{2^{k}}$,

$$
\left\|\sum_{i=1}^{2^{k}} c_{i} x_{s_{n_{i}}}\right\| \geq \delta \sum_{i=1}^{2^{k}}\left|c_{i}\right|
$$

Proof. Consider the set $\mathcal{A}$ of all chains $\beta=\left\{s_{1}<s_{2}<\ldots\right\} \in \mathcal{C}(S)$ which satisfy the following property: there is a $\delta>0$ such that for any $k \in \mathbb{N}$, any $k \leq n_{1}<$ $n_{2}<\ldots<n_{2^{k}}$ and any scalars $c_{1}, c_{2}, \ldots, c_{2^{k}},\left\|\sum_{i=1}^{2^{k}} c_{i} x_{s_{n_{i}}}\right\| \geq \delta \sum_{i=1}^{2^{k}}\left|c_{i}\right|$.

For each $k \in \mathbb{N}$ let us denote

$$
\begin{aligned}
& \mathbb{Q}^{(k)}=\left\{B \subset \mathbb{Q} \mid \operatorname{card}(B)=2^{k}\right\}, \\
& \mathbb{N}_{(k)}=\left\{A \subset \mathbb{N} \mid \operatorname{card}(A)=2^{k} \text { and } k \leq \min A\right\} .
\end{aligned}
$$

Clearly, the sets $\mathbb{Q}^{(k)}, \mathbb{N}_{(k)}$ are countable.

Claim. The set $\mathcal{A}$ is a Borel subset of $\mathcal{C}(S)$.

Indeed, observe that $\beta=\left\{s_{1}<s_{2}<\ldots\right\} \in \mathcal{A}$

$\Leftrightarrow$ there is a $\delta>0$ such that for any $k \in \mathbb{N}$, any $k \leq n_{1}<n_{2}<\ldots<n_{2^{k}}$ and any scalars $c_{1}, c_{2}, \ldots, c_{2^{k}},\left\|\sum_{i=1}^{2^{k}} c_{i} x_{s_{n_{i}}}\right\| \geq \delta \sum_{i=1}^{2^{k}}\left|c_{i}\right|$

$\Leftrightarrow$ there is a $q \in \mathbb{Q}^{+}$such that for any $k \in \mathbb{N}$, any $A=\left\{n_{1}<\ldots<n_{2^{k}}\right\} \in \mathbb{N}_{(k)}$ and any $B=\left\{c_{1}, \ldots, c_{2^{k}}\right\} \in \mathbb{Q}^{(k)},\left\|\sum_{i=1}^{2^{k}} c_{i} x_{s_{n_{i}}}\right\| \geq q \sum_{i=1}^{2^{k}}\left|c_{i}\right|$. Therefore,

$$
\mathcal{A}=\bigcup_{q \in \mathbb{Q}^{+}} \bigcap_{k \in \mathbb{N}} \bigcap_{A \in \mathbb{N}_{(k)}} \bigcap_{B \in \mathbb{Q}^{(k)}} \mathcal{D}_{q, k, A, B}
$$

where, if $A=\left\{n_{1}<n_{2}<\ldots<n_{2^{k}}\right\}$ and $B=\left\{c_{1}, c_{2} \ldots, c_{2^{k}}\right\}$,

$$
\mathcal{D}_{q, k, A, B}=\left\{\beta=\left\{s_{1}<s_{2}<\ldots\right\} \in \mathcal{C}(S)\left|\left\|\sum_{i=1}^{2^{k}} c_{i} x_{s_{n_{i}}}\right\| \geq q \sum_{i=1}^{2^{k}}\right| c_{i} \mid\right\} .
$$

Next we argue that $\mathcal{D}_{q, k, A, B}$ is an open subset of $\mathcal{C}(S)$. Let $\beta=\left\{s_{1}<s_{2}<\ldots\right\}$ belong to $\mathcal{D}_{q, k, A, B}$. We set

$$
U=\left\{\gamma=\left\{t_{1}<t_{2}<\ldots\right\} \in \mathcal{C}(S) \mid t_{j}=s_{j} \text { for } j=n_{1}, \ldots, n_{2^{k}}\right\} .
$$

Clearly, $U$ is an open neighborhood of $\beta$ in $\mathcal{C}(S)$. Moreover, for each $\gamma$ in $U$ we have

$$
\left\|\sum_{i=1}^{2^{k}} c_{i} x_{t_{n_{i}}}\right\|=\left\|\sum_{i=1}^{2^{k}} c_{i} x_{s_{n_{i}}}\right\| \geq \delta \sum_{i=1}^{2^{k}}\left|c_{i}\right| .
$$

Hence, $U \subset \mathcal{D}_{q, k, A, B}$. Thus the set $\mathcal{D}_{q, k, A, B}$ is open and the set $\mathcal{A}$ is Borel.

By Theorem [1.3 there is a subtree $S^{\prime}$ of $S$ such that either (a) $\mathcal{C}\left(S^{\prime}\right) \subset \mathcal{A}$ or (b) $\mathcal{C}\left(S^{\prime}\right) \cap \mathcal{A}=\emptyset$.

Now consider any chain $\beta$ of $S^{\prime}$. Then the sequence $\left(x_{s}\right)_{s \in \beta}$ is weakly null and no subsequence of $\left(x_{s}\right)_{s \in \beta}$ is Cesàro summable. By Theorem [1.2, there is a subchain $\alpha \subset \beta$ such that $\alpha \in \mathcal{A}$. Therefore, the case (b) is impossible and for the subtree $S^{\prime}$ we have $\mathcal{C}\left(S^{\prime}\right) \subset \mathcal{A}$. That is, for any chain $\beta$ of $S^{\prime}$ there is a $\delta>0$ such that 
for any $k \in \mathbb{N}$, any $k \leq n_{1}<n_{2}<\ldots<n_{2^{k}}$ and any scalars $c_{1}, c_{2}, \ldots, c_{2^{k}}$, $\left\|\sum_{i=1}^{2^{k}} c_{i} x_{s_{n_{i}}}\right\| \geq \delta \sum_{i=1}^{2^{k}}\left|c_{i}\right|$.

It remains to prove that we can find a subtree $T$ of $S^{\prime}$ such that the same $\delta>0$ works for any chain $\beta$ of $T$. To avoid introducing additional notation, we assume that for the original tree $S$ we have $\mathcal{C}(S) \subset \mathcal{A}$. Also let $\mathcal{M C}(S)$ denote the set of maximal chains of $S$.

Let $\left\{q_{n}\right\}_{n=1}^{\infty}$ be an enumeration of the positive rational numbers. For any $n \in \mathbb{N}$ we set

$$
\begin{array}{r}
\mathcal{A}_{n}=\left\{\beta=\left\{s_{1}<s_{2}<\ldots\right\} \in \mathcal{M C}(S)\left|\left\|\sum_{i=1}^{2^{k}} c_{i} x_{s_{n_{i}}}\right\| \geq q_{n} \sum_{i=1}^{2^{k}}\right| c_{i} \mid\right. \text { for any } \\
\left.k \in \mathbb{N}, k \leq n_{1}<\ldots<n_{2^{k}} \text { and any } c_{1}, \ldots, c_{2^{k}} \in \mathbb{R}\right\} .
\end{array}
$$

Clearly, $\mathcal{M C}(S)=\bigcup_{n=1}^{\infty} \mathcal{A}_{n}$. Since the set $\mathcal{M C}(S)$ has cardinality $2^{\omega}$, there is $n \in \mathbb{N}$ such that the set $\mathcal{A}_{n}$ has cardinality $2^{\omega}$.

For every node $s \in S$ we set

$$
B_{s}=\left\{\beta \in \mathcal{M C}(S) \mid \beta \in \mathcal{A}_{n} \text { and } s \in \beta\right\} .
$$

We next construct a subtree $T=\left\{t_{\emptyset}, t_{0}, t_{1}, t_{(0,0)}, \ldots\right\}$ of $S$. Let $t_{\emptyset}=\emptyset \in T$. The following claim contains the main argument for our construction.

Claim. There are noncomparable nodes $t_{0}, t_{1}$ such that each one of the sets $B_{t_{0}}, B_{t_{1}}$ has cardinality $2^{\omega}$.

Indeed, suppose that the assertion is not true. Then one of the sets $B_{0}, B_{1}$ has cardinality $2^{\omega}$ while the other has cardinality less than $2^{\omega}$. Without loss of generality, we can assume that $\operatorname{card}\left(B_{0}\right)=2^{\omega}$. Similarly, one of the sets $B_{(0,0)}, B_{(0,1)}$ has cardinality $2^{\omega}$ and the other one has cardinality less than $2^{\omega}$. Continuing in a similar way, we inductively construct a maximal chain $\beta=\left\{s_{0}=\emptyset<s_{1}<s_{2}<\ldots\right\}$ and a sequence $t_{1}, t_{2}, \ldots$ of nodes of $S$ such that for every $k$ the following properties hold:

(i) $\operatorname{lev}\left(t_{k}\right)=k$ and $s_{k-1}<t_{k}$,

(ii) $B_{t_{k}}$ has cardinality less than $2^{\omega}$,

(iii) $B_{s_{k}}$ has cardinality $2^{\omega}$.

Now observe that $\mathcal{A}_{n}=\bigcup_{k=1}^{\infty} B_{t_{k}} \cup\{\beta\}$. Consequently, at least one of the sets $B_{t_{k}}, k \in \mathbb{N}$, must have cardinality $2^{\omega}$, and we have reached a contradiction.

Therefore we can find noncomparable nodes $t_{0}, t_{1}$ such that each one of the sets $B_{t_{0}}, B_{t_{1}}$ has cardinality $2^{\omega}$. These nodes complete the first level of $T$.

Repeating the same argument, we find noncomparable nodes $t_{(0,0)}, t_{(0,1)}$ such that $t_{0}<t_{(0,0)}, t_{0}<t_{(0,1)}$ and each one of the sets $B_{t_{(0,0)}}, B_{t_{(0,1)}}$ has cardinality $2^{\omega}$. Hence, we inductively construct a subtree $T=\left\{t_{s} \mid s \in S\right\}$ such that for every $s \in S$, the set $B_{t_{s}}=\left\{\beta \in \mathcal{A}_{n} \mid t_{s} \in \beta\right\}$ has cardinality $2^{\omega}$.

Claim. The subtree $T$ has the desired property with $\delta$ equal to $q_{n}$.

Indeed, let $\beta=\left\{t_{1}<t_{2}<\ldots\right\}$ be a maximal chain of $T, k \in \mathbb{N}, k \leq n_{1}<n_{2}<$ $\ldots<n_{2^{k}}$ and $c_{1}, c_{2}, \ldots, c_{2^{k}} \in \mathbb{R}$. We show that

$$
\left\|\sum_{i=1}^{2^{k}} c_{i} x_{t_{n_{i}}}\right\| \geq \delta \sum_{i=1}^{2^{k}}\left|c_{i}\right| .
$$


By the construction of $T$, there is a maximal chain $\tilde{\beta}$ of $S$ such that $\tilde{\beta} \in \mathcal{A}_{n}$ and $t_{n_{2^{k}}} \in \tilde{\beta}$. Since $\tilde{\beta} \in \mathcal{A}_{n}$, the above inequality is verified easily.

Finally, if $\alpha$ is any chain of $T$, then there is a unique maximal chain $\beta$ of $T$ such that $\alpha \subset \beta$. Since the sequence $\left(x_{s}\right)_{s \in \beta}$ satisfies the desired property, so does the subsequence $\left(x_{s}\right)_{s \in \alpha}$.

For the next corollary, we need to recall some basic results concerning spreading models. The following theorem is due to A. Brunel and L. Sucheston [2], and its proof uses Ramsey's theorem.

Theorem 4.2. Let $\left(x_{n}\right)$ be a bounded sequence in the Banach space $X$. Then there exists a subsequence $\left(x_{n}^{\prime}\right)$ of $\left(x_{n}\right)$ such that for any $k \in \mathbb{N}$ and any scalars $a_{1}, \ldots, a_{k}$ the following limit exists:

$$
\lim _{\substack{n_{1}<\ldots<n_{k} \\ n_{1} \rightarrow \infty}}\left\|\sum_{i=1}^{k} a_{i} x_{n_{i}}^{\prime}\right\| .
$$

The limit given by the preceding theorem allows us to define a seminorm on the space $c_{00}$ of finitely supported sequences of real numbers by

$$
\left\|\sum_{i=1}^{k} a_{i} e_{i}\right\|=\lim _{\substack{n_{1}<<<<n_{k} \\ n_{1} \rightarrow \infty<}}\left\|\sum_{i=1}^{k} a_{i} x_{n_{i}}^{\prime}\right\| .
$$

Moreover, this seminorm defines a norm if and only if the sequence $\left(x_{n}^{\prime}\right)$ does not converge. In this situation, we set $F$ to be the completion of $c_{00}$ under the given norm. The space $F$ is called a spreading model of $X$ for the sequence $\left(x_{n}\right)$. Clearly, the sequence $\left(e_{i}\right)_{i=1}^{\infty}$ is spreading, which means that for all $n_{1}<n_{2}<\ldots<n_{k}$ and all scalars $a_{1}, a_{2}, \ldots, a_{k}$,

$$
\left\|\sum_{i=1}^{k} a_{i} e_{i}\right\|=\left\|\sum_{i=1}^{k} a_{i} e_{n_{i}}\right\| .
$$

We also note that a bounded sequence $\left(x_{n}\right)$ may contain many different subsequences $\left(x_{n}^{\prime}\right)$ satisfying Theorem 4.2, which may give very different spreading models.

The connection between Rosenthal's theorem and the notion of a spreading model was first suggested by L. Tzafriri (see [7) and was studied in full detail by B. Beauzamy (see [1]). The following corollary is a consequence of Theorem 4.1 (see [1]).

Corollary 4.1. Let $\left(x_{s}\right)_{s \in S}$ be a bounded sequence in the Banach space $X$. Suppose that for any chain $\beta$ of $S$ the sequence $\left(x_{s}\right)_{s \in \beta}$ is weakly null and it is not Cesàro summable. Then there exist a subtree $T$ of $S$ and a constant $C>0$ such that if $\beta$ is any chain of $T$ and $F$ is any spreading model for the sequence $\left(x_{s}\right)_{s \in \beta}$, then the sequence $\left(e_{n}\right)$ in $F$ is $C$-equivalent to the usual $l_{1}$-basis.

Proof. Let $T$ be the subtree given by Theorem 4.1. Let $\beta$ be any chain of $T$ and let $(F,\|\|$.$) be any spreading model for the sequence \left(x_{s}\right)_{s \in \beta}$. It is enough to show that for any $k \in \mathbb{N}$ and $a_{1}, \ldots, a_{k} \in \mathbb{R}$,

$$
\left\|\sum_{i=1}^{k} a_{i} e_{i}\right\| \geq \delta \sum_{i=1}^{k}\left|a_{i}\right| .
$$


Indeed, suppose that $\alpha=\left\{s_{1}<s_{2}<\ldots\right\}$ is a subchain of $\beta$ such that for any $k \in \mathbb{N}$ and $a_{1}, \ldots, a_{k} \in \mathbb{R}$,

$$
\left\|\sum_{i=1}^{k} a_{i} e_{i}\right\|=\lim _{\substack{n_{1}<\ldots<n_{k} \\ n_{1} \rightarrow \infty}}\left\|\sum_{i=1}^{k} a_{i} x_{s_{n_{i}}}\right\|
$$

Then the desired inequality follows immediately by Theorem 4.1.

\section{Spreading models of tree-SEquences}

In this section we generalize the theory of spreading models to tree-sequences. This enables us to give a stronger version of Corollary 4.1. First recall that using Ramsey theory we have (see [2])

Theorem 5.1. Suppose that $\left(x_{n}\right)$ is a normalized basic sequence in the Banach space $X$ and that $\left(\epsilon_{i}\right)$ is a decreasing sequence of positive real numbers converging to 0 . Then there is a subsequence $\left(x_{n}^{\prime}\right)$ of $\left(x_{n}\right)$ and a normalized basic sequence $\left(e_{n}\right)$ (not necessarily in $X$ ) such that

$$
\left|\left\|\sum_{i=1}^{2^{k}} a_{i} x_{n_{i}}\right\|-\left\|\sum_{i=1}^{2^{k}} a_{i} e_{i}\right\|\right|<\epsilon_{k}
$$

for any $k \in \mathbb{N}$, any $\left(a_{i}\right)_{i=1}^{2^{k}} \subset[-1,1]$ and any $k \leq n_{1}<\ldots<n_{2^{k}}$.

Using Stern's theorem, we generalize Theorem 5.1 to tree-sequences and obtain a uniformity of spreading model estimates on the chains.

Theorem 5.2. Let $\left(x_{s}\right)_{s \in S}$ be a normalized sequence in the Banach space X. Let $\left(\epsilon_{i}\right)$ be a decreasing sequence of positive real numbers converging to 0 . Then there is a subtree $T$ of $S$ such that for any maximal chain $\beta=\left(s_{i}\right)_{i=1}^{\infty}$ of $T$, any $k \in \mathbb{N}$, any $\left(a_{i}\right)_{i=1}^{2^{k}} \subset[-1,1]$, any $k \leq n_{1}<\cdots<n_{2^{k}}$ and any $k \leq m_{1}<\cdots<m_{2^{k}}$,

$$
\left|\left\|\sum_{i=1}^{2^{k}} a_{i} x_{s_{n_{i}}}\right\|-\left\|\sum_{i=1}^{2^{k}} a_{i} x_{s_{m_{i}}}\right\|\right| \leq \epsilon_{k} .
$$

Consequently, if for each such $\beta,\left(x_{s_{i}}\right)$ is basic, then the sequence $\left(x_{s}\right)_{s \in \beta}$ admits a spreading model $\left(e_{i}^{\beta}\right)_{i=1}^{\infty}$ satisfying

$$
\left|\left\|\sum_{i=1}^{2^{k}} a_{i} x_{s_{n_{i}}}\right\|-\left\|\sum_{i=1}^{2^{k}} a_{i} e_{i}^{\beta}\right\|\right| \leq \epsilon_{k}
$$

for any $k \in \mathbb{N},\left(a_{i}\right)_{i=1}^{2^{k}} \subset[-1,1]$, and $k \leq n_{1}<\cdots<n_{2^{k}}$.

Proof. Consider the set $\mathcal{A}$ of all chains $\beta=\left\{s_{1}<s_{2}<\cdots\right\} \in \mathcal{C}(S)$, which satisfy the following property: for any $k \in \mathbb{N}$, any $\left(a_{i}\right)_{i=1}^{2^{k}} \subset[-1,1]$, any $k \leq n_{1}<\cdots<n_{2^{k}}$ and any $k \leq m_{1}<\cdots<m_{2^{k}},\left|\left\|\sum_{i=1}^{2^{k}} a_{i} x_{s_{n_{i}}}\right\|-\left\|\sum_{i=1}^{2^{k}} a_{i} x_{s_{m_{i}}}\right\|\right| \leq \epsilon_{k}$.

Claim. The set $\mathcal{A}$ is a Borel subset of $\mathcal{C}(S)$. 
Indeed, we have:

$$
\begin{aligned}
& \beta=\left\{s_{1}<s_{2}<\cdots\right\} \in \mathcal{A} \\
& \Leftrightarrow \text { for any } k \in \mathbb{N} \text {, any }\left(a_{i}\right)_{i=1}^{2^{k}} \subset[-1,1] \text {, any } k \leq n_{1}<\cdots<n_{2^{k}} \text { and any } \\
& k \leq m_{1}<\cdots<m_{2^{k}},\left|\left\|\sum_{i=1}^{2^{k}} a_{i} x_{s_{n_{i}}}\right\|-\left\|\sum_{i=1}^{2^{k}} a_{i} x_{s_{m_{i}}}\right\|\right| \leq \epsilon_{k} \\
& \Leftrightarrow \text { for any } k \in \mathbb{N} \text {, any } B=\left\{q_{1}, \ldots, q_{2^{k}}\right\} \in \mathbb{Q}^{2^{k}} \cap[-1,1]^{2^{k}}, \text { any } A=\left\{n_{1}<\right. \\
& \left.\cdots<n_{2^{k}}\right\} \in \mathbb{N}(k) \text { and any } C=\left\{m_{1}<\cdots<m_{2^{k}}\right\} \in \mathbb{N}_{(k)}, \mid\left\|\sum_{i=1}^{2^{k}} q_{i} x_{s_{n_{i}}}\right\|- \\
& \left\|\sum_{i=1}^{2^{k}} q_{i} x_{s_{m_{i}}}\right\| \mid \leq \epsilon_{k} .
\end{aligned}
$$

Therefore

$$
\mathcal{A}=\bigcap_{k \in \mathbb{N}} \bigcap_{\substack{B \in \mathbb{Q}^{(k)} \\ B \subset[-1,1]}} \bigcap_{A, C \in \mathbb{N}_{(k)}} \mathcal{D}_{k, B, A, C}
$$

where, if $B=\left\{q_{1}, \ldots, q_{2^{k}}\right\}, A=\left\{n_{1}<\cdots<n_{2^{k}}\right\}$ and $C=\left\{m_{1}<\cdots<m_{2^{k}}\right\}$, then

$$
\mathcal{D}_{k, B, A, C}=\left\{\beta=\left\{s_{1}<s_{2}<\cdots\right\} \in \mathcal{C}(S)||\left\|\sum_{i=1}^{2^{k}} q_{i} x_{s_{n_{i}}}\right\|-\left\|\sum_{i=1}^{2^{k}} q_{i} x_{s_{m_{i}}}\right\| \mid \leq \epsilon_{k}\right\} .
$$

Clearly $\mathcal{D}_{k, B, A, C}$ is an open subset of $\mathcal{C}(S)$.

By Theorem 1.3, there is a subtree $T$ of $S$ such that either (a) $\mathcal{C}(T) \subset \mathcal{A}$ or (b) $\mathcal{C}(T) \cap \mathcal{A}=\emptyset$. By Theorem 5.1 we next must have (a).

Corollary 5.1. Let $\left(x_{s}\right)_{s \in S}$ be a normalized sequence in the Banach space $X$. Suppose that for any chain $\beta$ of $S$ the sequence $\left(x_{s}\right)_{s \in \beta}$ is weakly null and it is not Cesàro summable. Then there exist a subtree $T$ of $S$ and a constant $C>0$ such that for any chain $\beta=\left\{s_{1}<s_{2}<\cdots\right\}$ of $T$ for all $k \in \mathbb{N}$ and $k \leq n_{1}<\cdots<n_{k}$, $\left(x_{s_{n_{i}}}\right)_{i=1}^{k}$ is $C$-equivalent to the unit vector basis of $\ell_{1}^{k}$.

This follows from Corollary 4.1, Theorem 5.2 and the easily deduced fact that if $\left(x_{s}\right)_{s \in S}$ is a normalized sequence such that for all $\beta \in \mathcal{C}(S),\left(x_{s}\right)_{s \in \beta}$ is weakly null, then there exists a subtree $T$ of $S$ so that for all $\beta \in \mathcal{C}(T), \beta=\left(s_{i}\right),\left(x_{s_{i}}\right)_{i=1}^{\infty}$ is 2-basic.

\section{ACKNOWLEDGMENT}

The author would like to thank the referee for useful suggestions concerning Section 5 .

\section{REFERENCES}

1. B. Beauzamy, Banach-Saks properties and spreading models, Math. Scand. 44 (1979), 357-384. MR555227 (81a:46018)

2. A. Brunel and L. Sucheston, On B-convex Banach spaces, Math. System Theory 7 (1974), 294-299. MR0438085(55:11004)

3. N. Dunford and J.T. Schwartz, Linear operators. I: General theory, Pure and Appl. Math., vol. 7, Interscience, New York, 1958. MR0117523 (22:8302)

4. P. Erdös and M. Magidor, A note on regular methods of summability and the Banach-Saks property, Proc. Amer. Math. Soc. 59 (1976), 232-234. MR0430596 (55:3601)

5. F. Galvin and K. Prikry, Borel sets and Ramsey's theorem, J. Symbolic Logic 38 (1973), 193-198. MR0337630(49:2399)

6. A. Kechris, Classical Descriptive Set Theory, Springer, New York, 1995. MR1321597 (96e:03057) 
7. H. P. Rosenthal, Weakly independent sequences and the Banch-Saks property, in Proceedings of the Durham Symposium on the relations between infinite and finite dimensional convexity. Durham, July 1975, pp. 22-24.

8. J. Stern, A Ramsey theorem for trees with an application to Banach spaces, Israel J. Math. 29 (1978), 179-188. MR 0476554 (57:16114)

9. A. Tsarpalias, A note on the Ramsey property, Proc. Amer. Math. Soc. 127 (1999), 583-587. MR:1458267 (99c:04005)

Department of Mathematics, University of Athens, 15784, Athens, Greece

E-mail address: k-poulios@math.uoa.gr 